\title{
Detection of Bruises on Apples Using NeAr-Infrared HYPERSPECTRAL IMAGING
}

\author{
R. Lu
}

\begin{abstract}
Development of an automated bruise detection system will help the fruit industry to provide better fruit for the consumer and reduce potential economic losses. The objective of this research was to investigate the potential of near-infrared (NIR) hyperspectral imaging for detecting bruises on apples in the spectral region between $900 \mathrm{~nm}$ and $1700 \mathrm{~nm}$. An NIR hyperspectral imaging system was developed and a computer algorithm was created to detect both new and old bruises on apples. Experiments were conducted to acquire hyperspectral images from Red Delicious and Golden Delicious apples over a period of 47 days after bruising. Results showed that the spectral region between $1000 \mathrm{~nm}$ and $1340 \mathrm{~nm}$ was most appropriate for bruise detection. Bruise features changed over time from lower reflectance to higher reflectance, and the rate of the change varied with fruit and variety. Using both principal component and minimum noise fraction transforms, the system was able to detect both new and old bruises, with a correct detection rate from $62 \%$ to $88 \%$ for Red Delicious and from 59\% to 94\% for Golden Delicious. The optimal spectral resolution for bruise detection was between $8.6 \mathrm{~nm}$ and $17.3 \mathrm{~nm}$, with the corresponding number of spectral bands between 40 and 20. This research shows that NIR hyperspectral imaging is useful for detecting apple bruises. With improvement in image acquisition speed and detector technology, the NIR hyperspectral imaging technique will have the potential for offline inspection and online sorting of fruit for defects.
\end{abstract}

Keywords. Apple, Bruises, Defects, Fruit, Hyperspectral imaging, Near-infrared, Quality, Spectroscopy.

A pple bruises are of great concern to the apple industry and the retailer because they lower the quality grade of the fruit and can cause significant economic losses. Bruising normally happens to the tissue beneath the fruit skin. After the fruit tissue is damaged, its cells are initially filled with water and turn brownish. As time elapses, the damaged cells start to lose moisture and eventually become desiccated. It is a challenging task to detect bruises on the fruit because of the presence of fruit skin and because detection accuracies are affected by factors such as time, bruise type and severity, apple variety, and fruit pre- and post-harvest conditions.

A number of techniques have been researched for automated detection of bruises on apple fruit. Machine vision is widely used in the fruit industry to sort fruit for size and color. However, sorting for defects such as bruises using machine vision is still a challenging issue (Crowe and Delwiche, 1996). Commercial defect-sorting systems have become available recently, but their capability for detecting such subtle defects as bruises is still a problem. Research has found that near-infrared spectroscopy (NIRS) can be used to detect bruises on apples because freshly bruised tissue has lower reflectance than normal tissue in the visible and

Article was submitted for review in November 2001; approved for publication by Information \& Electrical Technologies Division of ASAE in November 2002.

Mention of commercial products is solely for providing factual information for the reader and does not imply endorsement by USDA.

The author is Renfu Lu, ASAE Member Engineer, Agricultural Engineer, USDA-ARS, 224 Farrall Hall, Michigan State University, East Lansing, MI 48824; phone: 517-432-8062; fax: 517-432-2892; e-mail: lur@msu.edu. shortwave near-infrared (Vis/NIR) region from $400 \mathrm{~nm}$ to 1000 nm (Brown et al., 1974; Geoola et al., 1994; Upchurch et al., 1990). However, since NIRS only measures a small area of tissue (or point measurement), it is not suitable for inspecting whole apples. Throop et al. (1995) used a line scan NIR camera to obtain images from whole apples and developed an image processing algorithm to detect both new and old bruises. The detection results varied greatly depending on bruise severity and the bruise shape factor used in the algorithm. Later, they developed a multi-spectral imaging system using four wavelengths to detect defects including bruises (Aneshansley et al., 1997; Throop et al., 1999). No detailed information was reported on the effectiveness of the system for bruise detection. Others (Schatzki et al., 1997) used $\mathrm{x}$-ray imaging to detect defects on apples; however, the technique does not appear suitable for inspection of whole fruit at the present. Wen and Tao (1998) applied a dual-camera technique, one NIR camera and one infrared camera, for detecting surface defects of fruit.

Recently, hyperspectral imaging has been investigated for quality and safety inspection of agricultural products (Lawrence et al., 2001b; Lu et al., 1999; Park et al., 2001). Lu et al. (1999) studied hyperspectral imaging for detecting bruises on three cultivars of apples in the spectral region between $450 \mathrm{~nm}$ and $900 \mathrm{~nm}$. They found that only the NIR portion between $700 \mathrm{~nm}$ and $900 \mathrm{~nm}$ was needed in detecting bruises. The technique could detect bruises on apples that were otherwise difficult to identify with other imaging techniques including multispectral imaging. So far, bruise detection has been primarily confined to the Vis/NIR region approximately from $400 \mathrm{~nm}$ to $1000 \mathrm{~nm}$. Studies have shown that an extended region beyond Vis/NIR is more effective in detecting internal quality of fruits (Lu et al., 2000; Moons et al., 1997). However, the potential of using imaging technolo- 
gy in the extended NIR region for detecting bruises and other defects has not been explored because no suitable NIR imaging devices were available until recently. With the most recent progress in the InGaAs area array detector technology, we can now investigate the potential of the hyperspectral imaging technique in the NIR region for detection of bruises on apples.

\section{OBJECTIVES}

The overall objective of this research was to study the potential of hyperspectral imaging in the spectral region between $900 \mathrm{~nm}$ and $1700 \mathrm{~nm}$ for detection of bruises on apples. The specific objectives were to:

- Develop a NIR hyperspectral imaging system covering the spectral region from $900 \mathrm{~nm}$ to $1700 \mathrm{~nm}$ for bruise detection.

- Develop computer algorithms to identify and segregate both new and old bruises from the normal tissue of apple fruit.

- Identify the spectral region and band resolution that are appropriate for detection of bruises.

\section{Methods And Procedure \\ SAMPle Preparation}

Red Delicious and Golden Delicious apples were hand picked on the Horticultural Farm south of Michigan State University's campus in East Lansing, Michigan, during the 2000 harvest season. Only those apples that were free from blemishes and bruises by visual inspection were selected for the study. A special device was built for inducing bruises on the test apples. The device had a steel rod $483 \mathrm{~mm}$ long as a pendulum arm, one end of which was attached to a pivot while the other end had a steel disk $38 \mathrm{~mm}$ in diameter and $13 \mathrm{~mm}$ in height, weighing 113 grams. The pendulum was mounted on a wooden board and rested horizontally. By lifting the pendulum to a pre-selected position (or height) and then releasing it, the steel disk would impact the fruit sitting on a wooden holder to generate a bruise on the fruit.

Forty fruit for each cultivar were selected for the bruise study. Each apple was bruised at three different locations around the equatorial line of the fruit with three pendulum positions $(127,229$, and $330 \mathrm{~mm})$. The three impact positions were selected based on preliminary tests and the reasoning that bruises to be generated should be in the range between $16 \mathrm{~mm}$ and $28 \mathrm{~mm}$, which correspond to the bruise allowances for three quality grades of apples as specified in the USDA apple grading standards (USDA Agricultural Marketing Service, 1978). A second purpose of using the three positions was to determine if the NIR hyperspectral imaging described below would be capable of detecting various sizes or degrees of bruises on each fruit. However, it was later found that bruise sizes varied greatly among fruits, even for the same bruising position, because individual apple fruit had quite different susceptibilities to bruising. Hence, in processing hyperspectral images, bruises for the three bruising positions were not separated into different classes. The average bruise size was equivalent to about $23 \mathrm{~mm}$ diameter circular area for the Red Delicious apples and 21 $\mathrm{mm}$ for the Golden Delicious apples, based on the estimated bruise areas from the images of the peeled apples. The actual bruise sizes varied from about $10 \mathrm{~mm}$ to $30 \mathrm{~mm}$.

\section{HyPERSPECTRAL ImAging SySTEM}

A hyperspectral imaging system, schematically shown in figure 1, was developed for the study. The system mainly consisted of an InGaAs area array camera (Sensors Unlimited, Princeton, N.J.) covering the spectral range between $900 \mathrm{~nm}$ and $1700 \mathrm{~nm}$, an imaging spectrograph (Specim, Finland) attached to the camera, a $25 \mathrm{~mm}$ focal length TV lens, a computer equipped with a frame-grabbing board for controlling the camera and acquiring image data, a quartz halogen lamp, and a specially designed bio-cone roller device for positioning the fruit for imaging. The InGaAs camera had an area array of $320 \times 240$ pixels and a quantum efficiency greater than $70 \%$ over the specified spectral region. The imaging spectrometer, with a slit size of $50 \mu \mathrm{m}$ and an F-number of 2.8, scanned the fruit one line a time.

As the light beam entered the spectrograph, it was dispersed into different directions according to wavelength while preserving its spatial information. The dispersed light was then mapped onto the InGaAs detector array, resulting in a two-dimensional image, one dimension representing the spectral axis and the other the spatial information for the scanning line. The spectral resolution for the system was $4.3 \mathrm{~nm}$. By scanning the entire surface of the fruit, a complete three-dimensional hyperspectral image cube was created, where two dimensions represented the spatial information and the third represented the spectral information. The DC quartz halogen lamp had an output power control to generate the appropriate illumination needed for hyperspectral imaging. Light was delivered through two fiber bundles to two line lamps positioned about $30^{\circ}$ from the vertical line. The roller device was controlled by a variable-speed motor for synchronization with the imaging system.

The hyperspectral imaging system was calibrated following the procedure described in $\mathrm{Lu}$ and Chen (1998). Lawrence et al. (2001a) has developed an elaborate calibration procedure for an imaging spectroscopy system.

\section{IMAGE ACQUISITION}

The fruit were removed from cold storage $\left(0^{\circ} \mathrm{C}\right)$ about 12 hours before imaging to allow them to reach room temperature. Hyperspectral images of individual fruit were acquired over a period of 47 days at various intervals to evaluate the changes of bruises with time. Hyperspectral images were first taken from bruise-free apples before the bruise study was started. These images were used as a reference to assist in identifying bruises on the images taken after the fruit were bruised. Within four hours after bruises were induced on the fruit, the first set of hyperspectral images

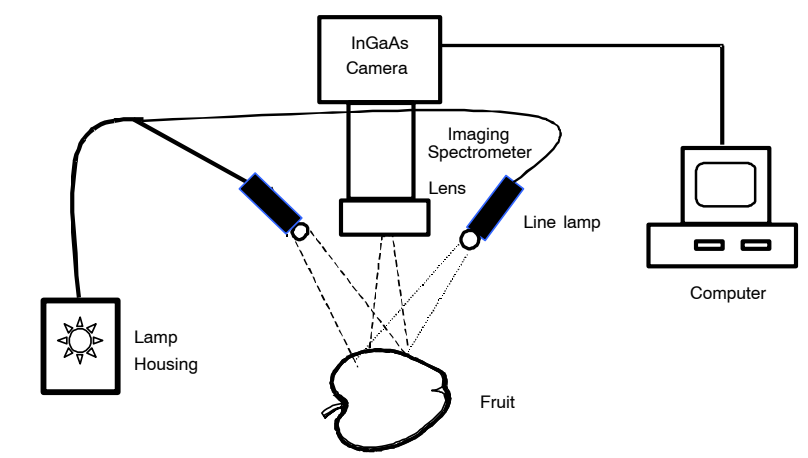

Figure 1. Schematic of the near-infrared hyperspectral imaging system for detecting bruises on apples. 
were taken from each fruit. The hyperspectral imaging system scanned each fruit at a speed of 12 frames/sec, and a total of 100 scans (approximately $9 \mathrm{~s}$ ) were acquired from each fruit. An in-house computer program was written in $\mathrm{C}++$ to control the system, acquire line scan images, and then convert the scanned images into a hyperspectral image file for each fruit. The hyperspectral image had a spatial resolution of $3 \mathrm{~mm}$ along the stem-calyx axis and $2 \mathrm{~mm}$ along the circumferential direction. The apples were imaged again at $1,5,12,19,33$, and 47 days after bruising to study the effect of time on bruises detection. These dates were determined based on previous studies of Lu et al. (1999) and Upchurch et al. (1994). Upchurch et al. (1994) reported that 24 hours after bruising, bruises were fully developed and showed a maximum reflectance contrast to normal tissue. They found that reflectance in the bruised tissue for Golden Delicious and Red Delicious continued to change over time in the first 30 days of bruising, and thereafter the reflectance change was minimal. During the imaging study, we also collected hyperspectral images from a Spectralon reflection panel, which has a refection efficiency of $98 \%$ or higher over the spectral region of interest. This image was used as a reference to correct the sample image, as described below. One dark current image (with no light entering the imaging spectrometer) was also acquired for every four apples.

\section{Bruise Detection Algorithm Algorithm Development}

Principal component transform (PC) and minimum noise fraction transform (MNF) are two commonly used techniques for extracting useful features from hyperspectral images. Both techniques are intended to maximize image features with a minimal number of independent components through orthogonal transformation of the original image data. The MNF transform further assumes that the original hyperspectral image contains both signal and noise, and by separating them before performing the transform, we can obtain improved image features. In this study, a computer program was developed using IDL (Research Systems, Boulder, Colo.), a structured programming language for image processing, to detect both new and old bruises. Before performing $\mathrm{PC}$ or $\mathrm{MNF}$ transform, the original sample images were corrected using the following equation:

$$
\mathrm{R}=\frac{(\text { sample image }- \text { dark image })}{(\text { reference image }- \text { dark image })} \times 1000
$$

where $\mathrm{R}$ is the relative reflectance, and the reference image was obtained from a Spectralon panel. The relative reflectance images were multiplied by 1000 so that the pixel value for the resultant images was between 0 and 1000, which was lower than the original (between 0 and 4091) but was sufficient for bruise detection. Once this procedure was completed, the corrected image was normalized using the following equation:

$$
\mathbf{R}_{\lambda}=\frac{\mathrm{R}_{\lambda}}{\sum \mathrm{R}_{\lambda} / \mathrm{N}}
$$

where $\lambda$ is the wavelength, and $\mathrm{N}$ is the number of pixels for the scanning line. The normalization procedure shown in equation 2 allowed us to eliminate or reduce the variations of reflectance in the image caused by illumination source and sample geometry (Polder et al., 2000).

After completing the above preprocessing steps, PC or MNF transform was applied to enhance bruise features and reduce data dimensionality. Bruises were normally present in the third MNF image, and they could appear as either dark or bright, not necessarily following the same pattern in the original, untransformed images. A pixel was considered as part of the dark area if its value was less than $\mathrm{D}=280$, and the same procedure was followed for identifying bright areas if the pixel value was greater than $\mathrm{W}=800$. The threshold values used in this study were determined based on the observation of bruise features obtained with our specific system configurations, which would be different if the system parameters were changed. Bruises normally appeared opposite to those in the original, untransformed hyperspectral images after PC transform of the first 40 bands; that is, bruises that appeared dark in the original image would turn bright on the first PC image, and vice versa. However, the bruise features from PC transform were often not as conspicuous as those shown from MNF transform.

To overcome the shortcomings associated with each transform method, an approach was developed to use both transforms for effective detection of bruises on apples. As such, both MNF and PC transforms were first performed on the original, untransformed images. The third MNF image was used for further features extraction. Before doing so, a low-pass filter was used to improve the image quality. The resultant image was then rescaled from the original range between 0 and 4091 to that between 0 and 1000. Further, a 4\% linear stretch was used to improve the image contrast. In the meantime, PC transform was also performed on the first half bands of the original hyperspectral images. Since the first PC image contained the most significant bruise features, it was used to assist in deciding whether the corresponding areas in the third MNF image, either dark or bright, were true or false bruises. The third MNF images normally contained both bright and dark areas. Either group could be a candidate for bruises since MNF did not give a pattern of bruises that was consistent with the original, untransformed image. Therefore, the first step was to locate and identify both bright and dark areas. The MNF image was classified into three groups: dark, bright, and a third group between dark and bright. The third group was from the areas of normal tissue and therefore was dropped from consideration. Once these locations were identified, the actual geometry of each location was determined by comparing the values of adjacent pixels with a pre-selected value. At this stage, we have identified all dark and white areas. The remaining task was to determine which group represents true bruises.

At first, only the first PC image was used to judge if the dark or bright areas were true bruises. The mean pixel values were calculated from the PC image for the areas that corresponded to the dark areas and the bright areas identified on the MNF image. The two means were then compared. The areas with a higher mean were considered bruises. This approach worked well when bruises were relatively new so that they would appear bright on the PC images. However, as bruises got older, they changed from darker to brighter in the original, untransformed images. As a result, the approach proposed was found to be ineffective in detecting old bruises or when mixed bruises, appearing either dark or bright, were present on the same fruit. The algorithm was therefore 


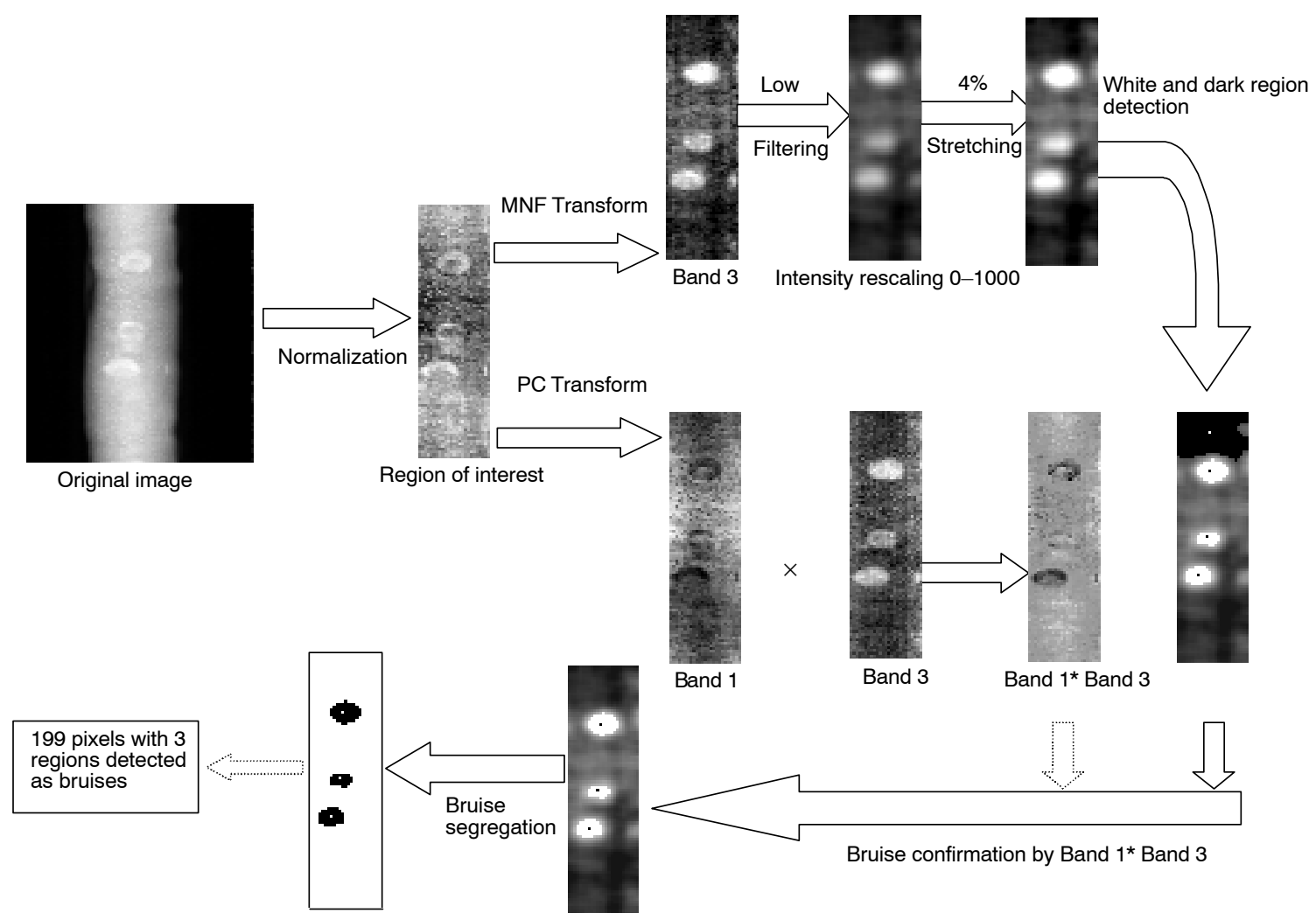

Figure 2. Flowchart showing the procedures used to detect bruises on apples.

modified to detect both old and new bruises. One important observation from the PC images was that bruises on the first and third PC images appeared just opposite. If bruises appeared dark (or bright) on the first PC image, then they appeared bright (or dark) on the third PC image. This pattern was found to be consistent regardless of bruise age. Therefore, in the modified bruise detection algorithm, a multiplication of the first and third PC images was performed to produce a new image, in which bruises, both old and new, would always appear to be darker than normal tissue. By comparing the mean pixel values for the two groups of areas corresponding to those identified in the MNF images, true bruises were identified. The procedures described above for detecting bruises are shown in figure 2 . It took about 2 to $3 \mathrm{~s}$ to complete the procedures shown in figure 2 with a $733 \mathrm{MHz}$ Pentium III computer. The main computational time was used for performing PC and MNF transforms.

\section{Spectral Resolution Selection}

In developing a hyperspectral imaging system, it is important that an optimal (or minimal) bandwidth or spectral resolution be used so that the amount of data to be processed is minimal, but without sacrificing detection results. To determine the optimal spectral resolution, binning, which is a process often used to reduce the number of pixels and increase the signal-to-noise ratio by summing up or averaging adjacent pixels in the image, was applied to reduce the total number of bands from 80 to $40,20,10$, and 5, with the corresponding spectral resolutions of 4.3, 8.6, 17.3, 34.6, and $69.2 \mathrm{~nm}$. The algorithm described above was then used to detect bruises for the two apple cultivars with different bruise ages. The final detection results were compared to quantify the effect of bands number or spectral resolution on detection results.

\section{Results AND Discussion}

Figure 3 shows typical reflectance spectra extracted from pixels of the hyperspectral images representing the normal (nonbruised) tissue and both new and old bruises from a Red Delicious and a Golden Delicious fruit over a spectral region between $900 \mathrm{~nm}$ and $1700 \mathrm{~nm}$. The reflectance from the new bruise (fig. 3a) was consistently lower than that from the normal tissue over the entire spectral region. The difference in reflectance was greatest in the region between $900 \mathrm{~nm}$ and $1400 \mathrm{~nm}$. Above $1400 \mathrm{~nm}$, the difference in reflectance between the bruised and nonbruised tissue decreased, and the spectral images had higher levels of noise with low reflectance. The reflectance of bruises changed over time and was affected by apple variety and fruit pre- and post-harvest conditions. An opposite pattern was observed for old bruises (fig. 3b), which had higher reflectance than normal tissue over the spectral region between 900 and $1400 \mathrm{~nm}$. Above $1400 \mathrm{~nm}$, the difference in reflectance between bruised and normal tissue decreased, and the trend was not consistent. These results show that the spectral region between 900 and $1400 \mathrm{~nm}$ would be appropriate for detecting both new and old bruises. However, since the noise level below $1000 \mathrm{~nm}$ and beyond $1340 \mathrm{~nm}$ increased considerably, the region between $1000 \mathrm{~nm}$ and $1340 \mathrm{~nm}$ appeared to be the most appropriate for bruise detection. This was further confirmed by comparing the results with those obtained with the entire spectral regions between $900 \mathrm{~nm}$ and $1700 \mathrm{~nm}$. 

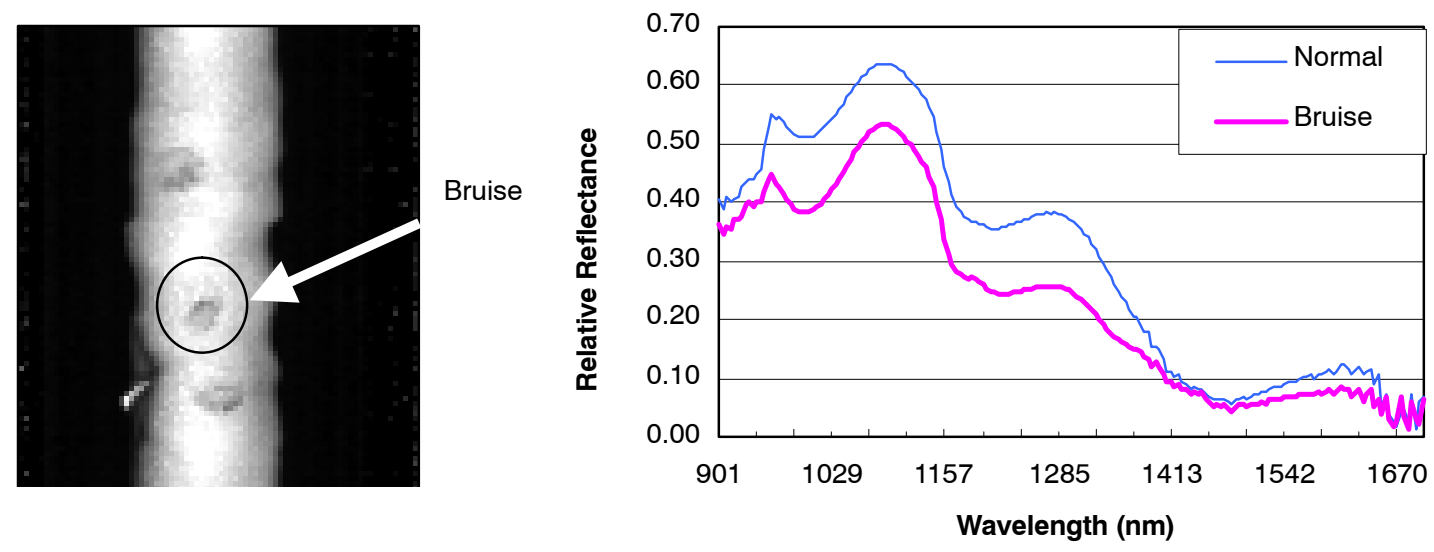

(a) Red Delicious
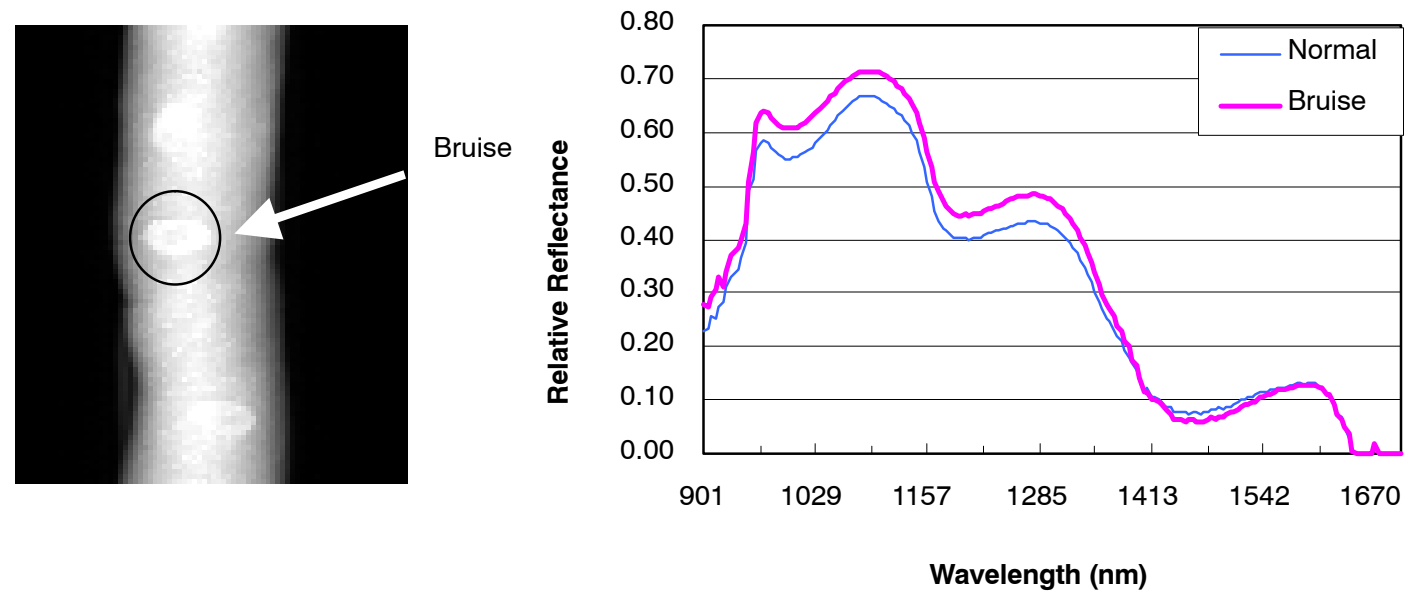

(b) Golden Delicious

Figure 3. NIR spectral images and typical spectral curves extracted from the selected pixels of the hyperspectral image representing normal and bruised tissue from: (a) a Red Delicious fruit 5 days after bruising, and (b) a Golden Delicious fruit 47 days after bruising.

\section{Time EFFECT ON BRUISES}

Figure 4 shows the changes in bruises with time from the spectral images at $1255 \mathrm{~nm}$ of a Red Delicious apple taken over a period of 47 days. Within hours after bruising, bruises were visible from the image but had not developed fully. One day after bruising, bruises had fully developed and appeared darker than normal tissue on the image. Bruises were most visible on the images taken at 5, 12, and 19 days. Thereafter, they started to change from dark to bright. At the last day (day 47), two bruises turned bright and one still remained dark. For Red Delicious apples tested in this study, most bruises appeared brighter than normal tissue at days 33 and 47 . A similar pattern of changes was found for Golden Delicious apples. However, the time at which most bruises turned from dark to bright was only about five days after bruising, considerably shorter than that for Red Delicious apples. These results indicate that bruises are affected by apple variety and bruise severity, and they change with time and at different rates, even for the same apple fruit. Hence, an effective detection system must have the capability to detect bruises, both new and old, for different apple cultivars.

\section{Bruise Detection}

Table 1 shows the results of bruise detection for Red Delicious apples for different days after bruising. As shown in the table, the correct detection rate, which is defined as the number of bruises identified from all apples with respect to the total number of actual bruises expressed in percentage, was low when bruises were less than about 4 hours old. The best detection results were obtained for Red Delicious apples one day after bruises were induced, with a correct detection rate of $88.1 \%$ and an incorrect detection rate of $11.2 \%$. The incorrect detection rate is the false classification of nonbruised tissue as bruised, expressed as a percentage of the total number of actual bruises from all test apples. As bruises became older, the correct classification rate decreased and was low at days 19 and 33, in which only $61.5 \%$ and $62.7 \%$ bruises were identified correctly. At day 47, the correct classification rate was improved to $68.6 \%$. This is because the bruised tissue continued to lose water and its reflectance increased as time elapsed, resulting in a larger difference in reflectance between normal and damaged tissue.

Table 2 shows the detection results for Golden Delicious apples. Contrary to the results for Red Delicious apples, the correct classification rates remained low at about $60 \%$ for days 0,1 , and 5 . Thereafter, the correct classification rates increased steadily and the best classification was obtained for the last day (day 47) with $94 \%$ bruises being correctly classified and only $7 \%$ incorrectly classified. The different trends of classification results for the two cultivars again 


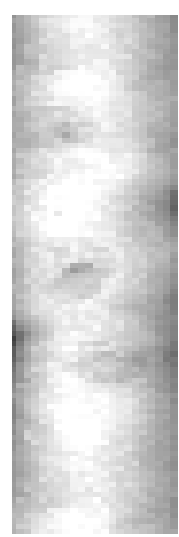

0 day

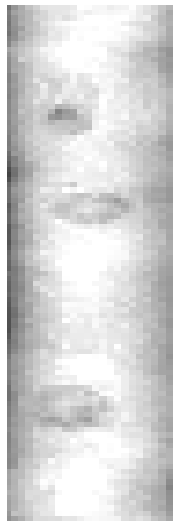

1 day

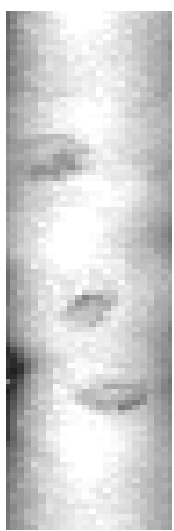

5 days

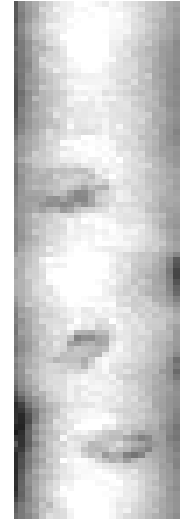

12 days

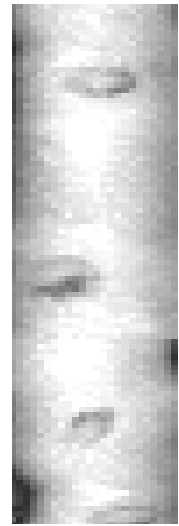

19 days

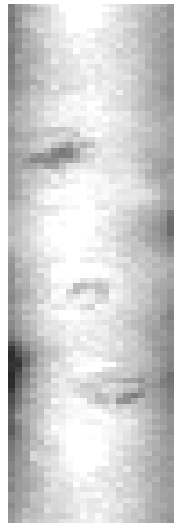

33 days

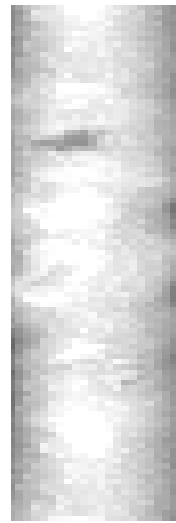

47 days

Figure 4. Spectral images at $1255 \mathbf{~ n m}$ for a Red Delicious apple showing the changes in bruises over time. There were three bruises on this particular fruit; the relative position of the bruises on each image may not be the same because the scanning did not necessarily start from the same location.

Table 1. Bruise detection results (in percent) for Red Delicious apples for different days after bruising. ${ }^{[a]}$

\begin{tabular}{lccccccc}
\hline & \multicolumn{7}{c}{ Days after Bruising } \\
\cline { 2 - 8 } & 0 & 1 & 5 & 12 & 19 & 33 & 47 \\
Correct rate & 69.6 & 88.1 & 84.1 & 70.7 & 61.5 & 62.7 & 68.6 \\
Incorrect rate & 23.0 & 11.2 & 7.9 & 19.5 & 21.0 & 27.7 & 22.2 \\
\hline
\end{tabular}

[a] The correct rate is defined as the percent of the number of bruises that were identified from all apples in terms of the total number of actual bruises. The incorrect or false rate is the percent of false classification of nonbruised areas as bruises in terms of the total number of actual bruises from all apples.

Table 2. Bruise detection results (in percent) for Golden Delicious apples for different days after bruising. ${ }^{[a]}$

\begin{tabular}{lccccccc}
\hline & \multicolumn{7}{c}{ Days After Bruising } \\
\cline { 2 - 8 } & 0 & 1 & 5 & 12 & 19 & 33 & 47 \\
Correct rate & 62.8 & 62.1 & 59.0 & 75.8 & 85.0 & 93.1 & 93.8 \\
Incorrect rate & 24.8 & 21.0 & 25.4 & 8.5 & 5.4 & 2.5 & 6.8 \\
\hline
\end{tabular}

[a] The correct rate is defined as the percent of the number of bruises that were identified from all apples in terms of the total number of actual bruises. The incorrect or false rate is the percent of false classifications of nonbruised areas as bruises in terms of the total number of actual bruises from all apples. indicate the different patterns of bruise changes over time. Higher correct classification rates for old bruises on Golden Delicious apples were attributed to the fact that the contrast between bruised and nonbruised tissue was greater as bruises became older, whereas for Red Delicious apples, the opposite was true.

Although the classification results were relatively low for selected days, they should be viewed in perspective. The results from this study are generally better than those reported in several previous studies using different imaging techniques (e.g., Throop et al., 1995; Crowe and Delwiche, 1996). In this study, bruises were induced using three different drop heights and they were not treated separately in our analyses. Since it is more difficult to detect less severe bruises than the severe ones, one would expect that detection results would be improved if less severe bruises had been separated from the more severe ones.

This study shows that NIR hyperspectral imaging can be used to detect bruises on apples. Comparing the results from this study with those reported by Lu et al. (1999), in which a back-illuminated CCD camera was used, it appears that both systems can be used to detect bruises on apples effectively. It would be interesting to evaluate the relative performance of the two imaging techniques on bruises detection. At 
Table 3. Comparison of bruise detection results (in percent) obtained using different spectral resolutions (the corresponding number of bands) for Red Delicious and Golden Delicious apples. ${ }^{[a]}$

\begin{tabular}{lccccc}
\hline & \multicolumn{5}{c}{$\begin{array}{c}\text { Spectral Resolution, nm } \\
\text { (Band Numbers) }\end{array}$} \\
\cline { 2 - 6 } & 4.3 & 8.6 & 17.3 & 34.6 & 69.2 \\
& $(79)$ & $(40)$ & $(20)$ & $(10)$ & $(5)$ \\
\hline Red Delicious & & & & \\
$\quad$ Correct & 61.2 & 88.1 & 88.1 & 82.1 & 65.7 \\
$\quad$ Incorrect & 22.4 & 11.2 & 11.9 & 17.2 & 32.1 \\
\hline Golden Delicious & & & & & \\
$\quad$ Correct & 87.6 & 93.8 & 91.9 & 85.7 & 29.2 \\
Incorrect & 8.1 & 6.8 & 6.2 & 10.6 & 50.3 \\
\hline
\end{tabular}

[a] The data for Red Delicious apples were taken one day after bruising, and the data for Golden Delicious were taken 47 days after bruising.

present, InGaAs imagers are more expensive and lower in image resolution than $\mathrm{CCD}$ imagers.

\section{SPECTRAL Resolution}

Table 3 shows the detection results for Red Delicious and Golden Delicious apples with different numbers of spectral resolutions or bands. The table indicates that the best classification results were obtained when the spectral resolution was $8.6 \mathrm{~nm}$ (corresponding to 40 spectral bands). The results from $17.3 \mathrm{~nm}$ spectral resolution (20 bands) were about the same as those from $8.6 \mathrm{~nm}$. When the spectral resolution was $34.6 \mathrm{~nm}$ or $69.2 \mathrm{~nm}$, the detection results became worse. Contrary to our expectation, the results from using $4.3 \mathrm{~nm}$ (80 bands) were not as good as those obtained with $8.6 \mathrm{~nm}$ (40 bands) and $17.3 \mathrm{~nm}$ (20 bands). This is because the binning process, which resulted in reduction in the spectral resolution, improved the image quality by increasing the signal-to-noise ratio. The results in table 3 show that the appropriate spectral resolution for detecting bruises using NIR hyperspectral imaging should be between 8.6 and $17.3 \mathrm{~nm}$. Bruise detection results will suffer when the spectral resolution is either too high or too low. This finding should be useful in development of an improved NIR hyperspectral imaging system and should require less data to be collected. This finding also provides useful guidance in determining the bandwidths of filters when a multi-spectral imaging system is considered.

\section{Conclusions}

This study found that the spectral region between $1000 \mathrm{~nm}$ and $1340 \mathrm{~nm}$ was most appropriate for detecting apple bruises. Bruise features changed over time from lower reflectance to higher reflectance, and the rate of the change was variety and fruit dependent. Consequently, detection results were also affected by the time after bruising. For Red Delicious apples, the best detection results were obtained when bruises were relatively new, with a correct classification rate up to $88 \%$ and an incorrect or false rate less than $12 \%$. For Golden Delicious apples, the best detections were obtained from old bruises, with a correct classification rate up to $94 \%$ and an incorrect rate less than $7 \%$. The spectral resolution between $17.3 \mathrm{~nm}$ and $8.6 \mathrm{~nm}$ offered the best bruise detection results for both apple cultivars.

NIR hyperspectral imaging can be used to detect bruises on apples effectively. Improvement in illumination is critical for acquiring quality images and should be considered in the future. At present, InGaAs detectors are still expensive and low in image resolution compared to CCD detector technology. Speed is the main constraint to the online application of hyperspectral imaging for detection of bruises and other defects because a large amount of data must be acquired and processed. With the latest development in hyperspectral imaging for simultaneous acquisition of three-dimensional hyperspectral image cubes, the speed problem could be eventually overcome.

\section{ACKNOWLEDGEMENT}

The author wishes to thank Mr. Jianguo Wang, a former graduate student in computer science at Michigan State University, for assisting in developing computer programs for this study. Thanks also go to Michigan State University's Horticulture Farm for providing test apples and to Dr. Randolph Beaudry in the Horticulture Department for allowing us to use their cold storage facilities for this study.

\section{REFERENCES}

Aneshansley, D., J. A. Throop, and B. L. Upchurch. 1997. Reflectance spectra of surface defects on apples. In Proc. Sensors for Nondestructive Testing International Conference, 143-160. Orlando, Fla. 18-21 February. Ithaca, N.Y.: NRAES-97.

Brown, G. K., L. J. Segerlind, and R. Summitt. 1974. Near-infrared reflectance of bruised apples. Trans. ASAE 17(1): 17-19.

Crowe, T. G., and M. J. Delwiche. 1996. Real-time defect detection in fruit - Part II: An algorithm and performance of a prototype system. Trans. ASAE 39(6): 2309-2317.

Geoola, F., F. Geoola, and U. M. Peiper. 1994. A spectrophotometric method for detecting surface bruises on 'Golden Delicious' apples. J. Agric. Eng. Res. 58(1): 47-51.

Lawrence, K. C., B. Park, W. R. Windham, and C. Mao. 2001a. Calibration of imaging spectrometry system for inspection of contaminated poultry carcasses. ASAE Paper No. 013129. St. Joseph, Mich.: ASAE.

Lawrence, K. C., W. R. Windham, B. Park, and R. J. Buhr. 2001 b. Hyperspectral imaging system for identification of fecal and ingesta contamination on poultry carcasses. ASAE Paper No. 013076. St. Joseph, Mich.: ASAE.

Lu, R., and Y. R. Chen. 1998. Hyperspectral imaging for safety inspection of food and agricultural products. SPIE 3544: 121-133.

Lu, R., Y. R. Chen, B. Park, and K-H. Choi. 1999. Hyperspectral imaging for detecting bruises in apples. ASAE Paper No. 993120. St. Joseph, Mich.: ASAE.

Lu, R., D. E. Guyer, and R. M. Beaudry. 2000. Determination of firmness and sugar content of apples using NIR diffuse reflectance. Journal of Texture Studies 31(6): 615-630.

Moons, E., P. Dardenne, A. Dubois, and M. Sindic. 1997. Nondestructive visible and NIR spectroscopy for the determination of internal quality in apple. In Proc. Sensors for Nondestructive Testing International Conference, 122-131. Orlando, Fla. 18-21 February. Ithaca, N.Y.: NRAES-97.

Park, B., K. C. Lawrence, W. R. Windham, and R. J. Buhr. 2001. Hyperspectral imaging for detecting fecal and ingesta contamination on poultry carcasses. ASAE Paper No. 013076. St. Joseph, Mich.: ASAE.

Polder, G., G. W. A. M. van der Heijden, and I. T. Young. 2000. Hyperspectral imaging analysis for measuring ripeness of tomatoes. ASAE Paper No. 003089. St. Joseph, Mich.: ASAE. Schatzki, T. F., R. P. Haff, R. Young, I. Can, I-C. Le, and N. Toyofuku. 1997. Defect detection in apples by means of x-ray imaging. Trans. ASAE 40(5): 1407-1415. 
Throop, J. A., D. J. Aneshansley, and B. L. Upchurch. 1995. An image processing algorithm to find new and old bruises. Trans. ASAE 11(5): 751-757.

Throop, J. A., D. J. Aneshansley, and B. Anger. 1999. Multispectral images for detecting defects on apples in real time. ASAE Paper No. 993205. St. Joseph, Mich.: ASAE.

Upchurch, B. L., H. A. Affeldt, W. R. Hruschka, K. H. Norris, and J. A. Throop. 1990. Spectrophotometric study of bruises on whole 'Red Delicious' apples. Trans. ASAE 33(2): 585-589.
Upchurch, B. L., J. A. Throop, and D. J. Aneshansley. 1994. Influence of time, bruise-type, and severity on near-infrared reflectance from apple surfaces for automatic bruise detection. Trans. ASAE 37(5): 1571-1575.

USDA Agricultural Marketing Service. 1978. Apples: Market inspection instructions. Washington, D.C.: U.S. Government Printing Office.

Wen, Z., and Y. Tao. 1998. Method of dual-camera NIR/MIR image for fruit sorting. ASAE Paper No. 983043. St. Joseph, Mich.: ASAE. 